\title{
In-situ production of Histamine-imprinted polymeric materials for electrochemical monitoring of fish
}

\author{
Verónica M. Serrano ${ }^{\mathrm{a}, \mathrm{b}, \mathrm{c}}$, Ana R. Cardoso ${ }^{\mathrm{a}, \mathrm{b}, \mathrm{c}}$, Mário Diniz ${ }^{\mathrm{d}}$, M. Goreti F. Sales ${ }^{\mathrm{a}, \mathrm{b}, \mathrm{c}, *}$

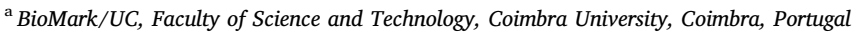 \\ ${ }^{\mathrm{b}}$ BioMark/ISEP, School of Engineering of the Polytechnic Institute of Porto, Porto, Portugal \\ ${ }^{\mathrm{c}}$ CEB, Centre of Biological Engineering, Minho University, Braga, Portugal \\ ${ }^{\mathrm{d}}$ UCIBIO-REQUIMTE, Faculty of Science and Technology, Nova University, Lisbon, Portugal
}

Keyworas:

Molecularly imprinted polymer

Electropolymerization

Screen-printed electrode

Aniline

Histamine

Fish

\begin{abstract}
A B S T R A C T
Histamine (HIS) is a major public health problem due to its toxic properties. High levels can cause a chronic toxicity as poisoning and can be used as a signal of food hygiene. Thus, a new electrochemical sensor for HIS detection in fish is presented herein, prepared by tailoring a molecularly imprinted polymer (MIP) sensing material on a gold screen-printed electrode (Au-SPEs), in which the polymeric film was generated in-situ. This film was obtained by electropolymerizing aniline under conditions that preserved the chemical structure of HIS. Raman spectroscopy followed the chemical changes occurring at each stage of the electrode modification.

The device performance was assessed by evaluating the changes in electron transfer properties of a standard redox probe $\left[\mathrm{Fe}(\mathrm{CN})_{6}\right]^{4-} /\left[\mathrm{Fe}(\mathrm{CN})_{6}\right]^{3-}$, by cyclic voltammetry $(\mathrm{CV})$ and electrochemical impedance spectroscopy (EIS). EIS was also used to calibrate the sensor, being the standard solutions prepared under different background media (electrolyte or a blank sample of fish extract). The device displayed a linear response from $500 \mathrm{nM}$ to $1 \mathrm{mM}$, with a limit of detection of $210 \mathrm{nM}$, and a selective behaviour against tyramine, another amine related to fish degradation. The sensing system was further employed to monitor the HIS content in samples in different time points of storage at ambient temperature. The obtained results were in agreement with the ELISA method, while offering more reproducible data.

In general, the optimized sensor allowed reproducible and accurate analysis of fish samples subject to degradation and was completely assembled in-situ, in a very simple and straightforward approach. The device is low cost and suitable for further adaptation to on-site analysis, as required in food control.
\end{abstract}

\section{Introduction}

Histamine (HIS) is a relevant biogenic amine which acts as a mediator in local hypersensitivity (called HIS intolerance or HIS poisoning). It is present in several foods, such as vegetables, fruit, fermented foods, but specially in fish [1,2], with established legal limits for human consumption. If its concentration is lower than $10 \mathrm{mg} / \mathrm{kg}$, it indicates that the fish is of good quality; concentrations higher than $30 \mathrm{mg} / \mathrm{kg}$ mean significant deterioration; and concentrations of $50 \mathrm{mg} / \mathrm{kg}$ or higher are evidence of decomposition [2,3]. Furthermore, scombroid poisoning from HIS is associated with some specific fish species such as tuna, mackerel, sardine, herring, and anchovy [4]. Thus, it suggests the need for development of fast and inexpensive methods for HIS determination in-situ.

Conventional methods employed to determine HIS are essentially chromatographic-based, thereby being restricted to laboratorial facilities and sometimes requiring sample derivatization, which is unsuitable for routine and on-site analysis of HIS [5]. Alternative methods include HIS biosensors, combining a biorecognition element with a suitable transduction scheme. In general, electrochemical-based biosensors offer high sensitivity and selectivity, simplicity, precision, rapid response and low cost of instrumentation [4-6].

There are many electrochemical biosensors developed to target HIS. In general, their main difference is related to the nature of the biorecognition element, from which enzymes or antibodies are highlighted. Table 1 lists several enzyme-based biosensors found in the literature, employing different enzymes, in different combinations and different electrode supports, established by a direct product detection or involving a secondary enzyme reaction. Table 2 lists the only antibody-based HIS biosensors found in the literature. In these, HIS detection is performed directly or in a competition assay, involving sometimes redox mediators. Overall, enzymes and antibodies are naturally derived

\footnotetext{
* Corresponding author at: School of Engineering of the Polytechnique School of Porto, R. Dr. António Bernardino de Almeida, 431, 4200-072 Porto, Portugal. E-mail address: goreti.sales@eq.uc.pt (M.G.F. Sales).
} 


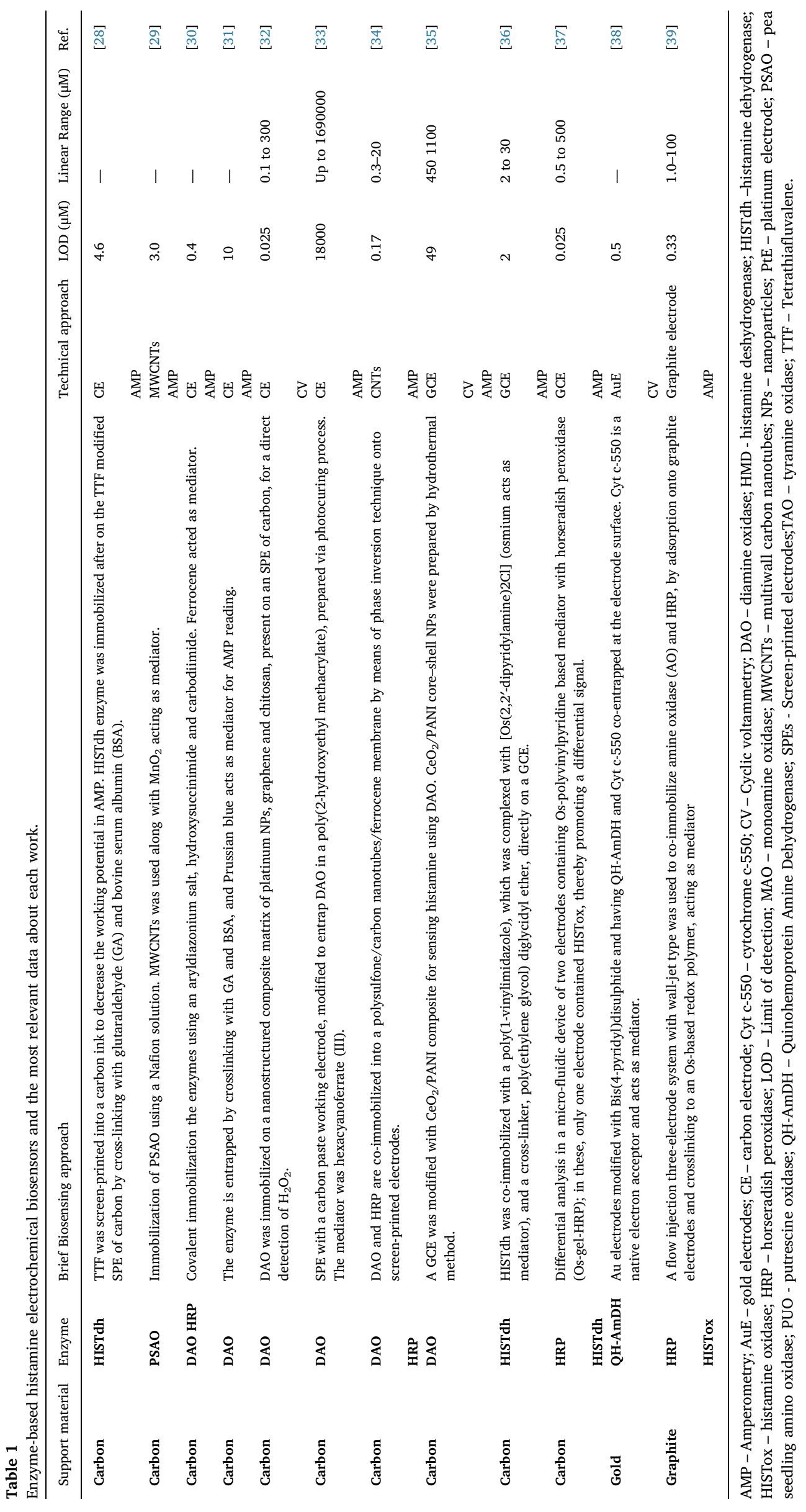


Table 2

Antibody-based histamine electrochemical biosensors and the most relevant data about each work.

\begin{tabular}{|c|c|c|c|c|c|}
\hline Support material & Brief Biosensing approach & $\begin{array}{l}\text { Technical } \\
\text { approach }\end{array}$ & LOD $(\mu \mathrm{M})$ & $\begin{array}{l}\text { Linear Range } \\
(\mu \mathrm{M})\end{array}$ & Ref. \\
\hline \multirow[t]{2}{*}{ Alumina } & $\begin{array}{l}\text { Magnetic NPs conjugated with His antibody were incubated in the sample for pre-concentration. } \\
\text { These conjugates were captured later in an alumina nanoporous membrane also containing His } \\
\text { antibody. Capturing the NPs resulted in a blocking effect monitored by EIS. }\end{array}$ & Magnetic NPs & 0.003 & $1.0-4000$ & [40] \\
\hline & & EIS & & & \\
\hline \multirow[t]{2}{*}{ GO } & $\begin{array}{l}\text { Graphene oxide on glass was modified with a His antibody to detect the presence of an His BSA } \\
\text { conjugate. }\end{array}$ & EIS & 0.1 & 0.1 to 1.0 & [41] \\
\hline & & SPR & & & \\
\hline Graphene & $\begin{array}{l}\text { His antibody was attached to a graphene surface. Then, the free histamine (from the sample) and } \\
\text { HRP tagged histamine molecules competed to bind to the antibodies. HRP further catalyses the } \\
\text { polymerization of 3,3-dimethoxybenzidine (PDB) in the presence of } \mathrm{H}_{2} \mathrm{O}_{2} \text { to produce the deposition } \\
\text { of an insulating PDB film, resulting in the decrease of the electrochemical current. The more } \\
\text { insulating, the less free histamine present in the sample. The redox mediator was ferricyanide. }\end{array}$ & SWV & 0.055 & $0.11-110$ & [42] \\
\hline
\end{tabular}

GO - graphene oxide; His - Histamine; HRP - horseradish peroxidase (HRP); NPs - nanoparticles; PDMS - polydimethylsiloxane; PBD - 3,3-dimethoxybenzidine; SWV - square-wave voltammetry; SPR - Surface Plasmon Resonance.

materials that display excellent selectivity features; however, they are also expensive and have poor stability under different conditions (humidity, temperature, $\mathrm{pH}$ and ionic content).

As an alternative to naturally-derived biorecognition elements, there are biomimetic materials such as molecularly-imprinted materials (MIPs) that may also offer high selectivity, rapid detection, and in-situ application feasibility [7]. In general, MIPs are synthetic materials prepared by polymerizing functional and cross-linking monomers around the target template, which is afterwards extracted to generate binding sites with complementary shape, size and functionalities [8]. Also, MIPs offer simple preparation, good stability, low cost, and robustness $[9,10]$.

Moreover, the selection of an electrical stimulus to initiate the polymerization is also an expeditous approach to produce MIP materials in-situ. Electrochemical techniques allow a strict control of the electrical parameters to be established at the electrode surface, thereby ensuring a strict control of the polymer growth. These are essential features to ensure the production of highly reproducible materials and, consequently, highly reproducible sensing devices. Yet, it is not a straightforward process when the target molecule itself undergoes oxidation under low potential values [11], which is the case of HIS. In this case, the careful selection of electrical and chemical conditions that allow a differential oxidation process between monomer and target molecule must be considered. Consequently, there are few MIP materials for HIS [12] and, as far as we know, there is only a single work producing the MIP by electropolymerization [13]. The later is a piezoelectric (acoustic) sensor that employs two distinct bis(bithiophene) derivatives as monomers, therefore it cannot be compared with electrochemical biosensors, especially in terms of cost and feasibility to perform analysis on-site.

Thus, as far as we know, this work presents for the first time an HIS electrochemical biosensor prepared in-situ, through the assembling of a MIP material with electropolymerized aniline. Aniline is used to make dyes and drugs, during the redox reaction and its polymerization yields polyaniline (PANI) [14]. PANI is a unique polymer, which has good electrical properties, good stability and reasonable cost [15]. Therefore, the MIP film was obtained by selecting the optimal conditions to establish a polyaniline-based imprinted film. The resulting biosensor was characterized and applied to determine HIS in fish samples (sardine and mackerel).

\section{Experimental section}

\subsection{Apparatus}

Electrochemical measurements were made by using a potentiostat/ galvanostat/impedance analyzer from PalmSens4, controlled by
PSTrace electrochemistry software. The Screen-Printed Electrodes (SPEs) were purchased from DropSens (DRP-220AT) and contained a silver reference electrode, a gold auxiliary electrode and a gold working electrode ( $4 \mathrm{~mm}$ diameter, Au-SPEs). Au-SPEs were linked to the potentiostat via a switch box produced by BioTID Electrónica.

\subsection{Reagents}

Ultrapure Milli-Q water was used throughout. All reagents were used as acquired, without further purification. Potassium hexacyanoferrate II-3-hydrate $\left(\mathrm{K}_{4}\left[\mathrm{Fe}(\mathrm{CN})_{6}\right] \cdot 3 \mathrm{H}_{2} \mathrm{O}\right)$ and potassium hexacyanoferrate III $\left(\mathrm{K}_{3}\left[\mathrm{Fe}(\mathrm{CN})_{6}\right]\right)$ were obtained from Riedel-deHäen; cysteamine chlorohidrate $\left(\mathrm{HSCH}_{2} \mathrm{CH}_{2} \mathrm{NH}_{2} \cdot \mathrm{HCl}\right)$ were purchased from Merck; HIS dihydrochloride, $\geq 99 \%$, lithium perchlorate and sulfuric acid 95-97\% $\left(\mathrm{H}_{2} \mathrm{SO}_{4}\right)$ were obtained from Sigma-Aldrich; aniline $\left(\mathrm{C}_{6} \mathrm{H}_{7} \mathrm{~N}\right)$ was obtained from Analar Normapur; phosphate buffered saline (PBS) tablets from Amresco; tyramine (TYR) from Sigma Aldrich.

\subsection{Solutions}

All solutions were prepared in ultrapure water. A $0.50 \mathrm{M} \mathrm{H}_{2} \mathrm{SO}_{4}$ solution was used to clean the commercial SPEs. A $0.05 \mathrm{M}$ cysteamine solution was prepared in ultrapure water. The MIP film was assembled with a solution of $0.01 \mathrm{M}$ HIS and $0.01 \mathrm{M}$ aniline, prepared in $0.20 \mathrm{M}$ lithium perchlorate (polymerization mixture). A non-imprinted polymer (NIP) material was prepared as control, using only $0.01 \mathrm{M}$ aniline in $0.20 \mathrm{M}$ of lithium perchlorate. The selectivity study compared the competitive behavior of a $10 \mu \mathrm{M}$ HIS solution, and a mixture of HIS and TYR with the same concentration $(10 \mu \mathrm{M})$, both prepared in $0.20 \mathrm{M}$ lithium perchlorate. HIS standard solutions used in the calibrations were also prepared in $0.20 \mathrm{M}$ lithium perchlorate and ranged from $1.0 \times 10^{-7}$ to $1.0 \times 10^{-2} \mathrm{M}$. The electrical changes occurring at the surface were followed by a solution of $5 \mathrm{mM}\left[\mathrm{Fe}(\mathrm{CN})_{6}\right]^{4-}$ and $[\mathrm{Fe}$ $\left.(\mathrm{CN})_{6}\right]^{3-}$, prepared in $0.1 \mathrm{M}$ PBS.

\subsection{Preparation of electrochemical biosensor on Au-SPE}

The working electrode of the Au-SPE was cleaned by electrochemical treatment, using $\mathrm{CV}$ from -0.1 at $1.5 \mathrm{~V}$, with a scan rate of $0.05 \mathrm{~V}$, for 5 cycles, in $0.50 \mathrm{M} \mathrm{H}_{2} \mathrm{SO}_{4}$. Afterwards, the gold surface was washed with ultrapure water. The next step consisted in the incubation of $0.05 \mathrm{M}$ Cysteamine, for $1 \mathrm{~h}$. The MIP layer was produced by chronoamperometry, at $+0.55 \mathrm{~V}$ for $150 \mathrm{~s}$, using the polymerization mixture. Finally, the template was removed by incubating the film in ultrapure water, for $15 \mathrm{~min}$. 

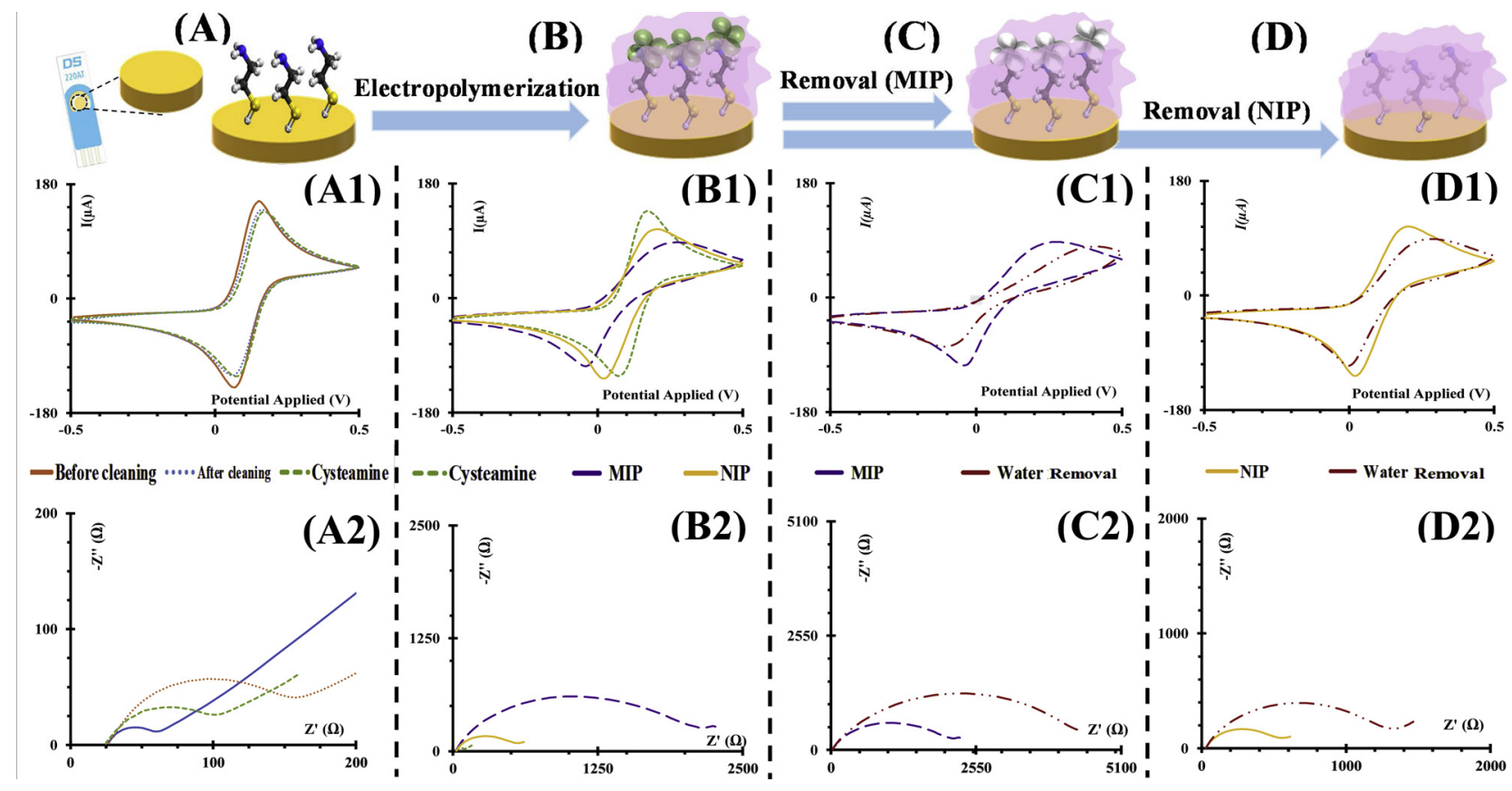

Fig. 1. Schematic illustration of electrochemical biosensor for detection of HIS (MIP and NIP): (A) Cleaning of Au/Cys; (B) Electropolymerization to produce Au/Cys/ $\mathrm{MIP}$ and $\mathrm{Au} / \mathrm{Cys} / \mathrm{NIP}$ surfaces; (C) template removal from the Au/Cys/MIP material; and (D) template removal procedure applied to the Au/Cys/NIP material.

\subsection{Raman analysis}

Raman spectroscopy analysis was used to follow each step of the MIP/NIP assembly. This was done by direct analysis of the material in a Thermo Scientific DXR Raman Spectroscope, equipped with a $785 \mathrm{~nm}$ laser. The average signal-to-noise ratio (peak height/RMS noise) was allowed for $900 \mathrm{~s}$, after $10 \mathrm{~min}$ photo bleaching, using a $1 \mathrm{~mW}$ laser power and a $50 \mu \mathrm{m}$ pinhole aperture.

\subsection{Electrochemical procedures}

All electrochemical assays were repeated three times. CV assays scanned potentials from -0.5 to $+0.5 \mathrm{~V}$, at $0.05 \mathrm{~V} / \mathrm{s}$, yielding information about redox potential and electrochemical reaction rates. EIS assays were performed at an open circuit potential, using a sinusoidal wave with an amplitude of $0.01 \mathrm{~V}$, and 50 data points, logarithmically distributed over $0.1-100000.0 \mathrm{~Hz}$ frequency range. The EIS data fitted a Randles equivalent circuit, using 5.5 PSTrace from PalmSens, and was analyzed by Nyquist plots, reflecting the mixed kinetic process taking place at the electrode-electrolyte interface that could be expressed as the real part of the impedance ( $\left.Z^{\prime}\right)$, which is the resistance, and its imaginary part (Z"). The charge-transfer resistance $\left(\mathrm{R}_{\mathrm{ct}}\right)$ was measured by the diameter of the semi-circle in the Nyquist plot. Square wave voltammetry (SWV) was also used, providing a sensitive and selective technique [16], and scanning potentials from -0.2 to $+0.8 \mathrm{~V}$.

The changes in the electrical properties of the sensing surface monitored the response of the redox probe solution. The limit of detection (LOD) was the concentration corresponding to $x+3 \sigma$, as extracted from the linear response, where $x$ was the average value of the blank signals and $\sigma$ the corresponding standard deviation [17]. For the selectivity studies, a competitive assay between HIS and a different biogenic amine usually found in fishery products was performed, using both molecules in the same concentration. In these studies, two independent devices were necessary to test the single HIS solution and the mixed solution of HIS and biogenic amine $(10 \mu \mathrm{M})$. The interfering specie selected for the assay was TYR [2].

\subsection{ELISA procedures}

Fish samples were also assessed for the content of HIS using an indirect Enzyme Linked Immunosorbent assay (ELISA). In brief, samples were coated $(50 \mu \mathrm{L} /$ well) on 96 -well microplates (Greiner Microlon, Germany) and allowed to incubate overnight, at $4{ }^{\circ} \mathrm{C}$. Then, the microplate was washed $(3 \times)$ in a washing solution (phosphate buffer solution with 0.05 Tween-20) and then blocked by adding $200 \mu \mathrm{L}$ of $1 \%$ BSA (Bovine Serum Albumin, Nzytech, Portugal). After $2 \mathrm{~h}$ incubation, the blocking solution was discharged, and the microplate washed $(3 \times)$ once again with the washing solution. Afterwards, a HIS monoclonal antibody (anti-HIS antibody, antibodies-online GmbH, Germany) was diluted to an appropriate concentration (1:200) and added to each well. The microplate was incubated at $37{ }^{\circ} \mathrm{C}$ for $90 \mathrm{~min}$. After another washing step, the secondary antibody (anti-mouse IgC, fc specific, conjugated for alkaline phosphatase (Sigma-Aldrich, Germany) was diluted (1:1000 in 1\% BSA) and $50 \mu$ ladded to each well followed by another incubation stage $\left(37^{\circ} \mathrm{C}\right.$ for $\left.90 \mathrm{~min}\right)$. After washing, $50 \mu \mathrm{l}$ of $p$ nitrophenyl phosphate substrate solution (PNPP tablets, Sigma-Aldrich) was added to each well and incubated for $30 \mathrm{~min}$ at room temperature. Then, $100 \mu \mathrm{l}$ of $3 \mathrm{M} \mathrm{NaOH}$ stop solution was added to each microplate well and the absorbance was read using a microplate reader at $405 \mathrm{~nm}$ (Biotek Synergy HTX, USA). For quantification, HIS standards were prepared by serial dilutions of purified carp HIS (Histamine analytical standard, Sigma-Aldrich, Germany) to give a range from 20 to $2000 \mathrm{ng}$ / $\mathrm{mL}$.

\section{Results and discussion}

\subsection{Electrode pre-treatment}

The practical use of SPEs requires their previous cleaning, in order to ensure reproducible electrochemical features among different units. For this purpose, three different treatments for cleaning the gold surface were tested: (i) cleaning the surface with absolute ethanol $(2 \times)$; (ii) cleaning with absolute ethanol $(2 \times)$ followed by electrochemical CV cleaning with a sulfuric acid solution; (iii) or using only an electrochemical stage, by $\mathrm{CV}$, using the same sulfuric acid solution. The 
potential applied in electrochemical cleaning and its duration were also optimized in previous assays, suggesting the favorable use of -0.1 to $1.5 \mathrm{~V}$, along $5 \mathrm{CV}$ cycles. The condition selected for further electrode cleaning was electrochemical cleaning, considering the resulting $\mathrm{R}_{\mathrm{ct}}$ decrease in the Nyquist plot and the increasing electrode reproducibility among different SPE units. Typical CV and EIS plots obtained under this condition are shown in Fig. 1A.

Before the MIP assembly, the clean gold electrode was modified to generate an amine layer. This amine layer would participate in the electropolymerization of aniline through its aromatic amine groups, thereby ensuring that the MIP film would be stably linked to the electrode layer. Cysteamine was used for this purpose, binding to the gold layer through its thiol group, - $\mathrm{SH}$, and leaving the amine groups exposed to the outer surface [18]. As expected, the addition of cysteamine contributed to an $R_{c t}$ increase, although of small significance when compared to the clean electrode (Fig. 1A). The resulting surface is identified by $\mathrm{Au} / \mathrm{Cys}$.

\subsection{Selecting the electrochemical conditions for polymerization}

The production of the MIP layer by electropolymerization requires the formation of radical species from the monomer compounds, by applying specific electrical conditions at the electrode surface. To ensure that the polymerization taking place at the electrode was not involving HIS, it was necessary to ensure that the selected electrical conditions would not affect the structure of HIS. If that happened, HIS would co-polymerize with aniline and stay permanently bounded to the polymeric layer, thereby limiting the formation of binding sites.

In this regard, the electrochemical features of aniline and HIS were first studied in single solutions and after in mixed solutions. The first tests allowed confirming that HIS and aniline were both electroactive in aqueous solutions in similar potential ranges. Thus, other background media were tested trying to identify a condition in which HIS would be inactive under an applied potential and aniline would remain electroactive under the same potential range. To this end, HIS solutions were prepared in $0.20 \mathrm{M} \mathrm{ACN}, 0.20 \mathrm{M}$ lithium perchlorate and $0.20 \mathrm{M}$ DMSO and electrochemically tested. The results obtained are shown in Fig. S1. In general, it was found that HIS was no longer electroactive within -1.0 to $+1.0 \mathrm{~V}$ when lithium perchlorate was used as dissolution medium (verified for concentrations up to $0.01 \mathrm{M} \mathrm{HIS}$ ). In contrast, the electroactivity of aniline persisted under the other conditions under study. Thus, using a lithium perchlorate medium would ensure the possibility of forming a PANI film without altering the structure of HIS.

\subsection{Assembly of the sensing layer}

The MIP film was obtained by electropolymerization, in which specific electrical conditions were applied to a solution containing both monomer (aniline) and template (HIS). This was done by bulk polymerization, which is the most common imprinting approach for producing MIP materials for small size target molecules. Bulk polymerization was combined herein with electropolymerization, which is an advantageous approach, when considering the short time required for polymerization to take place and the reduced reagent consumption. In addition, when compared to surface imprinting, this approach also ensures that imprinted sites are aways present on the outer layer of the biosensor [11,19,20].

In this, a pre-polymer arrangement is allowed to be formed between HIS and aniline, which is expected to involve hydrogen bond interactions. After this step, the polymeric network is formed by a radical reaction, initiated by giving enough potential to generate oxidized radicals of aniline. In agreement with the data in Fig. S2, a chrono-amperometric procedure involving the application of a potential of $+0.55 \mathrm{~V}$, for $150 \mathrm{~s}$, was established. The MIP electrode (Au/Cys/MIP) was obtained by electropolymerizing a mixed solution of aniline and HIS in lithium perchlorate, forming a film around the template, while the NIP electrode (Au/Cys/NIP, the control) was produced by using a solution of Aniline with lithium perchlorate, therefore the film was produced without template.

The resulting CV and EIS data is shown in Fig. 1B. Overall, the presence of the resulting polymeric layer was confirmed by the peak current decrease of the standard redox probe in the cyclic voltammogram (Fig. 1-B1) and the $\mathrm{R}_{\mathrm{ct}}$ increase in the Nyquist plot (Fig. 1-B2), when compared to the Au/Cys surface.

Moreover, there was a significant difference between MIP and NIP electrodes, which reflected the effect of the presence of HIS in the MIP material, because this was the single experimental difference in both materials. The $\mathrm{CV}$ assays of the Au/Cys/MIP electrode after chronoamperometry showed much lower peak currents and higher peak separation than the Au/Cys/NIP electrode (Fig. 1-B1). Consistently, the $R_{c t}$ value was much higher in the MIP film, thereby confirming the presence of HIS within the polymeric network. (Fig. 1-B1). Overall, the presence of HIS on the growing polymer changed the electrical properties of the surface at the time of polymer film growth. This could reflect the non-conductive properties of HIS itself and/or the change of the electrical features of the PANI film formed therein, especially because the conductive features of the PANI films is intrinsically linked to the conditions established for its polymerization [21]. In addition, the formation of less conductive polymeric layers in the first stages of the MIP polymerization contributed to the formation of less radicals per unit time at the external surface, and the rate of polymer formation decreased as the polymer was growing.

The reproducibility of independent electrodes was further tested and presented in Fig. 2. Summarizing, the CV and EIS data confirmed the great reproducibility of the electrochemical events, considering that 2-3 independent electrodes of Au/Cys/MIP (Fig. 2A) and Au/Cys/NIP (Fig. 2B) were involved herein and that each Au-SPE is intrinsically different as purchased.

\subsection{Removal of HIS from the sensing layer}

The final step of the MIP assembly was to remove the HIS template from the polymeric network. By removing HIS, binding sites of complementary shape to the target molecule would be formed within the polymer [22,23]. Since HIS is highly soluble in water, it is a good solvent to remove the molecule from the imprinted surface, thereby avoiding the use of other reagents that could alter intrinsic characteristics or damage the PANI layer.

The efficiency of the HIS removal was tested after incubating MIP and NIP films in water, for $15 \mathrm{~min}$. The $\mathrm{R}_{\mathrm{ct}}$ values in the Nyquist increased and the peak currents in the $\mathrm{CV}$ data decreased, both in NIP (Fig. 1C) and MIP films (Fig. 1D). Considering the NIP alone, this behavior could be linked to the exit of conductive oligomeric structures of PANI from the polymeric network, which is also something that could have happened in the MIP film. As the MIP showed a more intense effect than the NIP, this could reflect the additional exit of HIS from the polymeric network. In addition, HIS is positively charged under the test conditions, thereby establishing ionic interactions with the negatively charged iron redox probe and contributing to improve the chargetransfer properties at the electrode surface when it is there. Yet, the effect of the exit of HIS from the polymeric network is a balance between its non-conductive features and its ionic charge, and therefore its impact upon the Rct may vary.

Overall, these results confirmed an effective removal of HIS form the MIP films (when compared to the NIP) and a great stability of the PANI film when exposed to water.

\subsection{Morphological characterization of the biosensor}

Raman spectroscopy with a $785 \mathrm{~nm}$ laser gives valuable chemical information about gold materials and allows following-up their subsequent chemical modification. Raman spectra has been collected at 


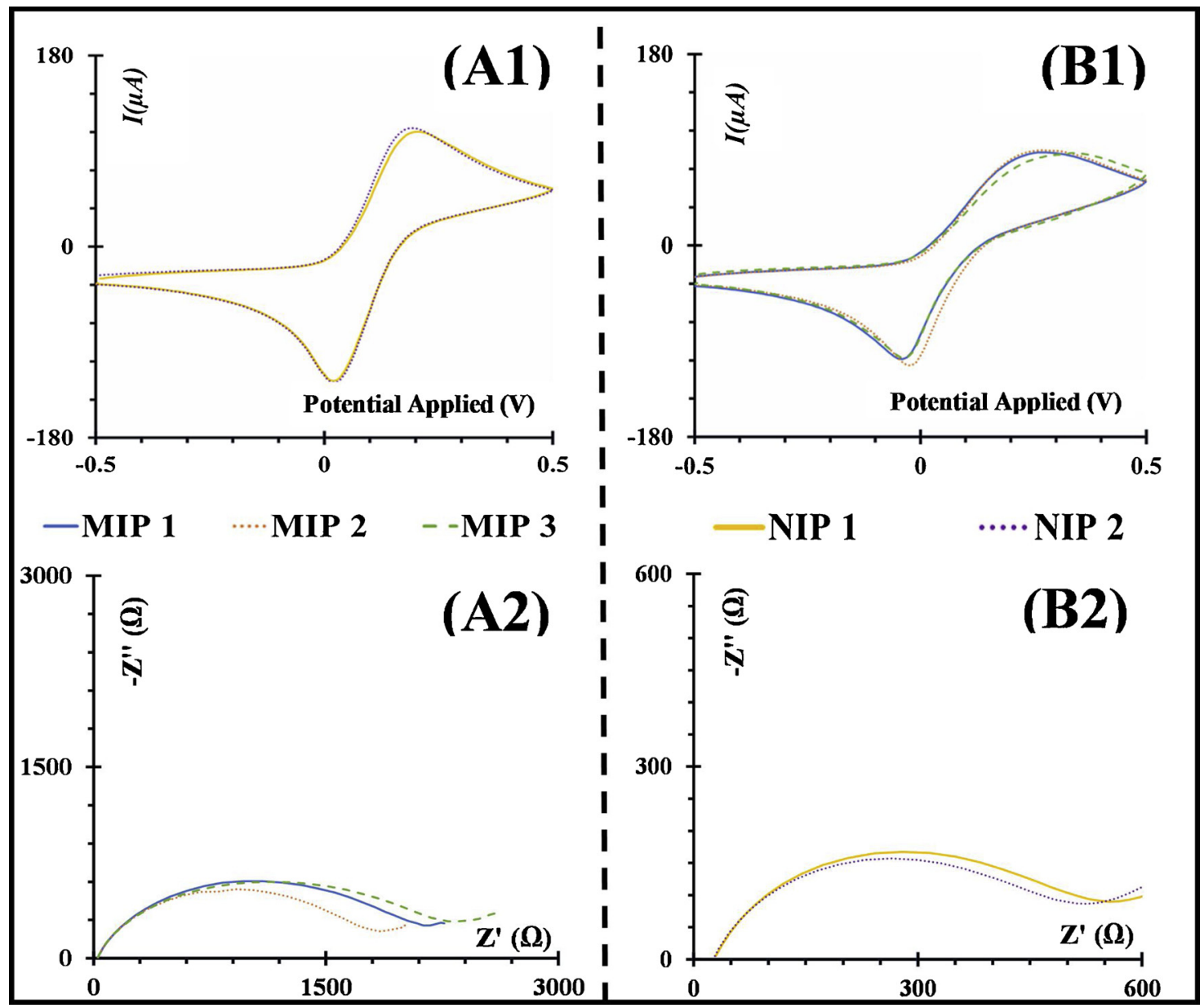

Fig. 2. Electrochemical readings of independent devices with (A) Au/Cys/MIP and (B) Au/Cys/NIP surfaces, in the form of (1) CV or (2) EIS data.

different stages of the biosensor assembly and shown Fig. S3, including a clean Au-SPE (Fig. S3 A) for comparison purposes. When cysteamine was added to the clean gold surface, new peaks were found at 719 e 685 $\mathrm{cm}^{-1}$ (Fig. S3-B), likely correlated to the $\mathrm{C}-\mathrm{S}$ stretching vibration and the conformation of Cys on the gold support [24,25]. The next step was the electropolymerization of the MIP (Fig. S3-C), yielding changes in the Raman shift of the maximum intensity peak, shifting to higher values, up to $575.51 \mathrm{~cm}^{-1}$. In addition, the two new peaks signaling cysteamine decreased their intensity, most likely being uncovered by the presence of the polymeric film. After the template removal (Fig. S3-D), the Raman shift of the maximum peak decreased to $560.82 \mathrm{~cm}^{-1}$, and the peaks signaling cysteamine were partially recovered, probably evidencing the exit of the HIS from the sensing surface. Overall, the Raman data confirmed the several modifications made to the Au-SPEs.

\subsection{Main analytical features}

The main analytical features of the Au/Cys/MIP and Au/Cys/NIP electrodes were evaluated by incubating first a drop of HIS standard solution on the working electrode for $20 \mathrm{~min}$ (to allow HIS binding), and by reading after the EIS electrical features of a standard iron redox probe casted on the three-electrode system. This was repeated for (i) increasing HIS concentrations to quantify the behavior of these electrodes, over a wide concentration range, and for (ii) independent electrodes (using a minimum of 3 ).

The behavior of the Au/Cys/MIP electrodes is presented in Fig. 3A, showing a typical Nyquist plot with blank solution and HIS concentrations ranging from $100 \mathrm{nM}$ to $1 \mathrm{mM}$ (left), prepared in lithium perchlorate, and the corresponding calibration curve, plotting absolute
$\mathrm{R}_{\mathrm{ct}}$ values against log concentration of HIS. The calibration plot evidences error bars that corresponded to three independent sensors, with three independent calibrations, thereby confirming the excellent reproducibility of the analytical system. The linear trend was observed from $500 \mathrm{nM}$ and $1 \mathrm{mM}$, with an average slope of $1992.2 \Omega /$ decade. The minimum squared correlation coefficient of all calibrations was 0.9957 , and the average limit of detection was $210 \mathrm{nM}$. The corresponding $\mathrm{Au}$ / Cys/NIP evaluations are also shown (Fig. 3B) and evidence a more random response against increasing HIS concentrations, thereby confirming the existence of non-specific binding of HIS to the PANI surface.

In general, increasing concentrations of HIS yielded increasing $\mathrm{R}_{\mathrm{ct}}$ values with a linear behavior within a wide range of concentrations. This behavior revealed mostly the non-conductive properties of HIS, which were probably dominating the effect of the cationic charge of HIS. This was probably related to the higher concentrations of HIS reached at the surface in the calibration procedure, when compared to the template removal stage.

In addition, the response of the biosensor was found stable, when considering that consecutive incubations of the blank solutions did not lead to visible alterations in the Nyquist plots. The corresponding Bode plots are also shown in Fig. 6.

\subsection{Selectivity test}

Selectivity evaluations were made by means of a competitive assay against other biogenic amine that could be present in fish. TYR was considered herein, because it is a typical interfering species for HIS readings $[2,26]$. This assay involved two independent sensing units and compared the effect of a single solution of $10 \mu \mathrm{M}$ HIS and that of a 


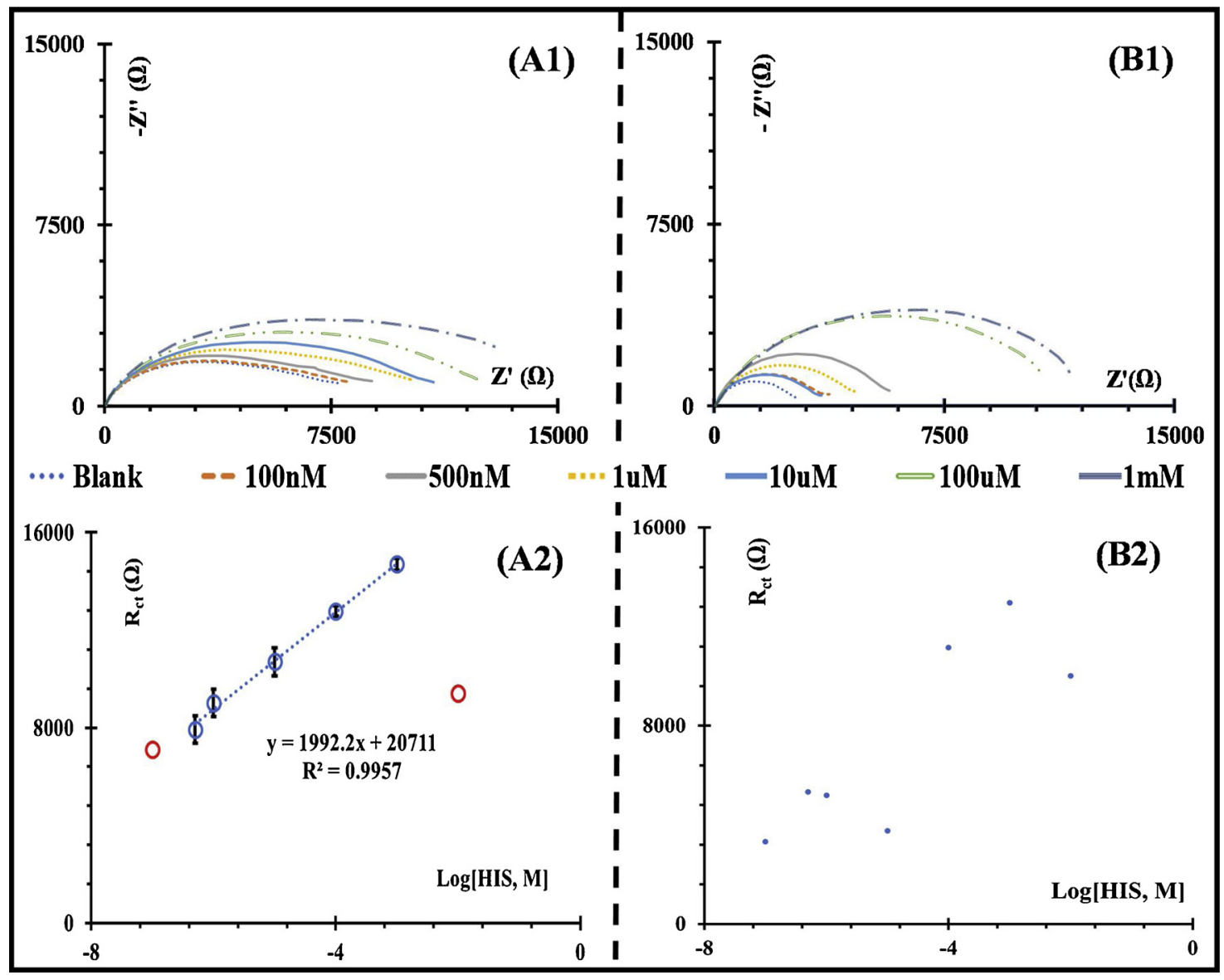

Fig. 3. EIS Au/Cys/MIP (A1) and Au/Cys/NIP (B1) sensor, and the corresponding calibration curves (A2 and B2).

mixed solution of $10 \mu \mathrm{M}$ HIS and $10 \mu \mathrm{M}$ TYR. It consisted in collecting first the signal of the blank (lithium perchlorate) in both sensing units and after the signal generated by the incubation of the single solution of HIS (in one sensing unit) and the mixed solution of HIS and TYR (in another sensing unit).

Fig. 4A shows the Nyquist plots of the Au/Cys/MIP electrodes evaluated under these conditions. The average percentage deviation upon the direct readings of HIS produced by TYR was $+1.59 \%$ (Fig. 4B). Overall, these results confirmed that TYR had a negligible interfering effect upon the HIS response and that the Au/Cys/MIP device was selective in the presence of other biogenic amine.

\subsection{Samples analysis}

As proof-of-concept, Au/Cys/MIP devices were applied to the analysis of real samples. Two different fish samples, sardine and mackerel, were used for this purpose. These samples were acquired in a supermarket, kept at room temperature to allow the formation of biogenic amines, and then frozen at three different timings $(0 \mathrm{~h} ; 12 \mathrm{~h}$ and $24 \mathrm{~h}$ ), [2]. Considering that the expected concentrations were unknown, two

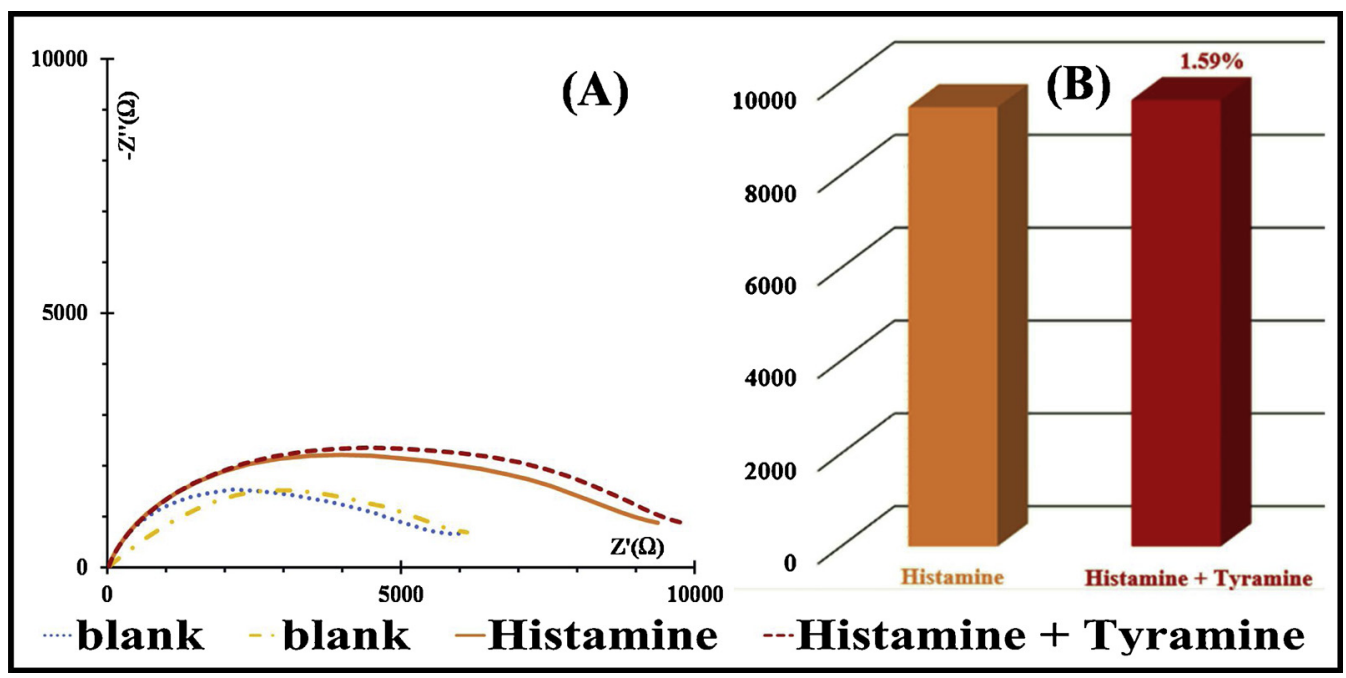

Fig. 4. Selectivity behavior of the biosensor for HIS $\left(1.00 \times 10^{-5} \mathrm{M}\right)$ against TYR $\left(1.00 \times 10^{-5} \mathrm{M}\right)$. 


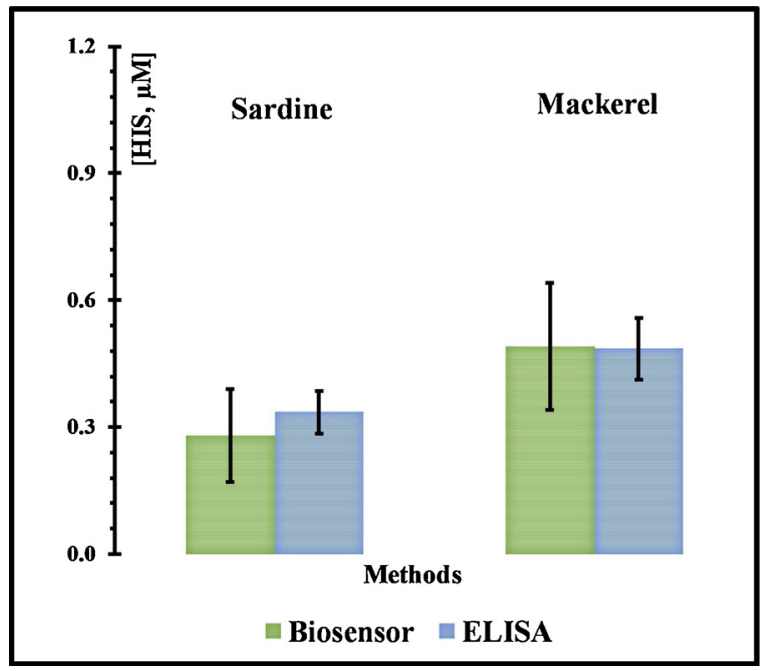

Fig. 5. Histamine concentration of sardine and mackerel samples (at $12 \mathrm{~h}$ ) obtained by the Au/Cys/MIP sensor and by an ELISA commercial kit.

different dilutions were used (1:1000 and 1:100) to check the biosensor response. These samples were also diluted in water, targeting the future direct analysis of samples, without any pre-treating procedures.

In general, it was clear that the increasing time lead to an increasing amount of HIS in the samples, which was already expected due to the occurrence of fish degradation at ambient temperature. A total of $12 \mathrm{~h}$ was enough to promote a significant increase of the HIS concentration in the sardine, while in the Mackerel samples a continuous increase was observed throughout time, being more significant for the $24 \mathrm{~h}$. Moreover, the lower dilution factor in both samples was always linked to higher $\mathrm{R}_{\mathrm{ct}}$ values, thereby confirming their higher concentration in HIS.

The real concentration of HIS in these samples was found by the known multiple standard addition method, as the samples were spiked with known and increasing amounts of HIS. These tests were made for samples exposed for $12 \mathrm{~h}$ to ambient temperature, as these were still below the typical linear response of the biosensors. Thus, the background (real) concentration in the samples was calculated by interacting with the standard addition method for a logarithm response in $x$ axis [27]. The experimental data obtained is shown in Fig. S4 and represents the spiked samples with known concentrations of HIS, using water as blank $(t=12 \mathrm{~h})$ for each type of fish. Considering the reading of three independent sardine samples, the calculated concentration was $2.8 \times 10^{-7}\left( \pm 1.1 \times 10^{-7}\right) \mathrm{M}$. The corresponding procedure involving mackerel samples yielded average values of $4.9 \times 10^{-7}$ $\left( \pm 1.5 \times 10^{-7}\right) \mathrm{M}$. In general, these results agreed with the direct sample readings, considering that the sardine samples had higher HIS concentrations, regardless the time of exposure to ambient temperature.

For further validation purposes, a commercial ELISA kit was used to analyze the same samples $(t=12 \mathrm{~h})$. The results obtained are plotted in Fig. 5. In detail, the average values obtained for the sardine samples were $3.3 \times 10^{-7}\left( \pm 0.6 \times 10^{-7}\right) \mathrm{M}$ and for the mackerel samples were $4.9 \times 10^{-7}\left( \pm 0.7 \times 10^{-7}\right) \mathrm{M}$. These results were in full agreement with the data generated by the Au/Cys/MIP devices.

Overall, the results obtained demonstrated that the electrode was selective and able to detect HIS concentrations, even when the concentrations of HIS in the samples were below the linear response range of the biosensor. The biosensor device may be further employed to follow-up fish degradation and the formation of biogenic amines.

\section{Conclusions}

The present work demonstrated the possibility of assembling a HIS biosensing device employing quick, expeditious and low-cost procedures. The most challenging step of this work was related with the electroactivity of the target molecule, which was solved by selecting experimental conditions that reduced the electroactivity features of HIS, while maintaining the electroactive properties of the monomer ANI. Generally, the biorecognition element was produced in-situ, within few seconds, and the on-site detection of HIS in aquatic environment was also allowed, requiring only a twenty-minutes incubation period.

In terms of analytical performance, the $\mathrm{Au} / \mathrm{Cys} / \mathrm{MIP}$ device displayed excellent analytical features, demonstrating high sensitivity over a wide range of linear responses, good selectivity against another competing biogenic amine, and the ability to be applied for real samples analysis. The device may be further employed to follow-up fish degradation and the formation of biogenic amines, which may be an interesting approach for food safety purposes.

\section{CRediT authorship contribution statement}

Verónica M. Serrano: Investigation, Data curation, Methodology, Validation, Writing - original draft. Ana R. Cardoso: Investigation, Supervision, Data curation, Methodology, Validation, Writing - original draft. Mário Diniz: Project administration, Validation, Funding acquisition. M. Goreti F. Sales: Conceptualization, Supervision, Resources,

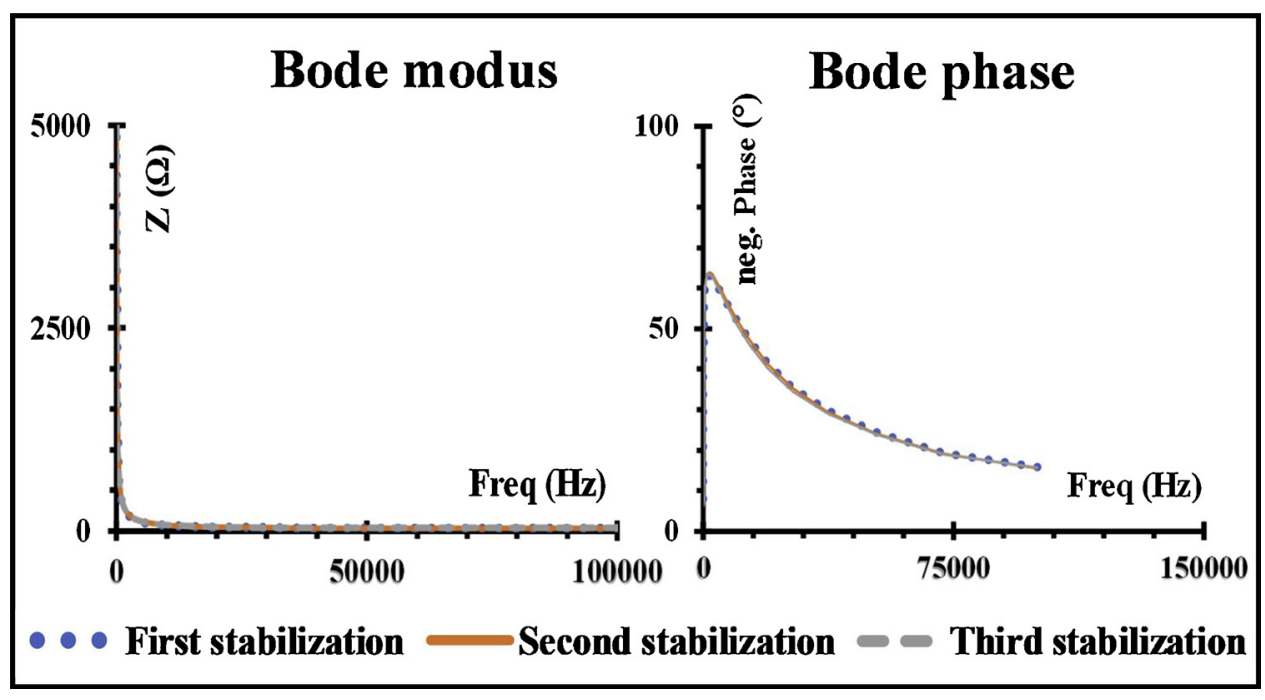

Fig. 6. Stability of the biosensor for HIS using Bode plots. 
Formal analysis, Writing - review \& editing.

\section{Declaration of Competing Interest}

The authors declare that they have no known competing financial interests or personal relationships that could have appeared to influence the work reported in this paper.

\section{Acknowledgements}

The authors acknowledge national funding to the National Foundation for Science and Technology (FCT), I.P., and European funding to FEDER (European Funding or Regional Development), via COMPETE2020 - POCI (operational program for internationalization and competitiveness), through projects PTDC/MAR-BIO/6044/2014 and PTDC/AAG-TEC/5400/2014.

\section{Appendix A. Supplementary data}

Supplementary material related to this article can be found, in the online version, at doi:https://doi.org/10.1016/j.snb.2020.127902.

\section{References}

[1] A. Balan, R. Cristescu, I. Stamatin, C. Nichita, A.C. Popescu, D.B. Chrisey, A.A. Ciucu, A.-M. Iordache, S.M. Iordache, E. Fagadar-Cosma, Histamine detection using functionalized porphyrin as electrochemical mediator, Comptes Rendus Chim. 21 (2017) 270-276, https://doi.org/10.1016/j.crci.2017.05.008.

[2] P. Taylor, L. Prester, Biogenic amines in fish, fish products and shellfish, Food Addit. Contam. (2011) 37-41.

[3] A. Veseli, M. Vasjari, T. Arbneshi, A. Hajrizi, ubomír Švorc, A. Samphao, K. Kalcher, Electrochemical determination of histamine in fish sauce using heterogeneous carbon electrodes modified with rhenium(IV) oxide, Sens. Actuators B Chem. 228 (2016) 774-781, https://doi.org/10.1016/j.snb.2016.01.085.

[4] M.M.G. Niraj, S. Pandey, Histamine biosensor: a review, Int. J. Pharm. Sci. Res. 3 (2012) 4158-4168, https://doi.org/10.13040/IJPSR.0975-8232.3(11).4158-2268.

[5] A. Geto, M. Tessema, S. Admassie, Determination of histamine in fish muscle at multi-walled carbon nanotubes coated conducting polymer modified glassy carbon electrode, Synth. Met. 191 (2014) 135-140, https://doi.org/10.1016/j.synthmet. 2014.03.005.

[6] S.K. Krishnan, E. Singh, P. Singh, M. Meyyappan, H.S. Nalwa, A review on graphene-based nanocomposites for electrochemical and fluorescent biosensors, RSC Adv. 9 (2019) 8778-8881, https://doi.org/10.1039/C8RA09577A.

[7] E. Bongaers, J. Alenus, F. Horemans, A. Weustenraed, L. Lutsen, D. Vanderzande, T.J. Cleij, F.J. Troost, R.J. Brummer, P. Wagner, A MIP-based biomimetic sensor for the impedimetric detection of histamine in different $\mathrm{pH}$ environments, Phys. Status Solidi Appl. Mater. Sci. 207 (2010) 837-843, https://doi.org/10.1002/pssa. 200983307.

[8] F. Barahona, B. Albero, J.L. Tadeo, A. Martín-Esteban, Molecularly imprinted polymer-hollow fiber microextraction of hydrophilic fluoroquinolone antibiotics in environmental waters and urine samples, J. Chromatogr. A 1587 (2019) 42-49, https://doi.org/10.1016/j.chroma.2018.12.015.

[9] M. Akhoundian, A. Rüter, S. Shinde, Ultratrace detection of histamine using a molecularly-imprinted polymer-based voltammetric sensor, Sensors (Switzerland) (2017) 1-10, https://doi.org/10.3390/s17030645.

[10] K.M.A. El-Nour, E.T.A. Salam, H.M. Soliman, A.S. Orabi, Gold nanoparticles as a direct and rapid sensor for sensitive analytical detection of biogenic amines, Nanoscale Res. Lett. 12 (2017), https://doi.org/10.1186/s11671-017-2014-z.

[11] A.R. Cardoso, A.P.M. Tavares, M.G.F. Sales, In-situ generated molecularly imprinted material for chloramphenicol electrochemical sensing in waters down to the nanomolar level, Sens. Actuators B. Chem. 256 (2018) 420-428, https://doi.org/10. 1016/j.snb.2017.10.114.

[12] F. Horemans, J. Alenus, E. Bongaers, A. Weustenraed, R. Thoelen, J. Duchateau, L. Lutsen, D. Vanderzande, P. Wagner, T.J. Cleij, MIP-based sensor platforms for the detection of histamine in the nano- and micromolar range in aqueous media, Sens. Actuators B. Chem. 148 (2010) 392-398, https://doi.org/10.1016/j.snb.2010.05. 003.

[13] A. Pietrzyk, S. Suriyanarayanan, W. Kutner, R. Chitta, F.D. Souza, Using a recognition film of the molecularly imprinted polymer of bis (bithiophene) derivatives, Analytical 81 (2009) 2633-2643.

[14] P. Tharmaraj, K. Pandian, K. Chitra, S. Devi, S. Meenakshi, Aniline-mediated synthesis of carboxymethyl cellulose protected silver nanoparticles modified electrode for the differential pulse anodic stripping voltammetry detection of mercury at trace level, Ionics (Kiel) 25 (2019) 3431-3441, https://doi.org/10.1007/s11581019-02858-0.

[15] E. Pashai, G.D. Najafpour, M. Jahanshahi, M. Rahimnejad, Highly sensitive amperometric sensor based on gold nanoparticles polyaniline electrochemically reduced graphene oxide nanocomposite for detection of nitric oxide, Int. J. Eng. 31
(2018) 188-195, https://doi.org/10.5829/ije.2018.31.02b.01.

[16] A. Chen, B. Shah, Electrochemical sensing and biosensing based on square wave voltammetry, Anal. Methods 5 (2013) 2158-2173, https://doi.org/10.1039/ c3ay40155c.

[17] D.A. Armbruster, T. Pry, Limit of Blank, Limit of Detection and Limit of Quantitation 29 (2008), pp. 49-52 doi:citeulike-article-id:3416410.

[18] F.T.C. Moreira, R.A.F. Dutra, J.P.C. Noronha, J.C.S. Fernandes, M.G.F. Sales, Novel biosensing device for point-of-care applications with plastic antibodies grown on Au-screen printed electrodes, Sens. Actuators B Chem. 182 (2013) 733-740, https://doi.org/10.1016/j.snb.2013.03.099.

[19] Y. Kobayashi, Y. Nakamitsu, Y. Zheng, Y. Takashima, H. Yamaguchi, A. Harada, Preparation of cyclodextrin-based porous polymeric membrane by bulk polymerization of ethyl acrylate in the presence of cyclodextrin, Polymer (Guildf.) 177 (2019) 208-213, https://doi.org/10.1016/j.polymer.2019.06.015.

[20] AbdAl-Rhman Magdy, Abdullah Youssef, Solution \& bulk polymerization written, Encycl. Dict. Polym. (2019), https://doi.org/10.1007/978-1-4419-6247-8_10882 677-677.

[21] C. Dhand, M. Das, M. Datta, B.D. Malhotra, Recent advances in polyaniline based biosensors, Biosens. Bioelectron. 26 (2011) 2811-2821, https://doi.org/10.1016/j. bios.2010.10.017.

[22] M.F. Frasco, L.A.A.N.A. Truta, M.G.F. Sales, F.T.C. Moreira, Imprinting technology in electrochemical biomimetic sensors, Sensors 17 (2017), https://doi.org/10. $3390 /$ s17030523.

[23] M. Peeters, Molecularly imprinted polymers (mips) for bioanalytical sensors: strategies for incorporation of mips into sensing platforms, Austin J. Biosens. Bioelectron. 1 (2015) 1-5.

[24] Q. Ma, H. Zhang, W. Liu, J. Ge, J. Wu, S. Wang, P. Wang, Surface-enhanced Raman scattering substrate based on cysteamine-modified gold nanoparticle aggregation for highly sensitive pentachlorophenol detection, RSC Adv. 6 (2016) 85285-85292, https://doi.org/10.1039/c6ra15774b.

[25] A. Abbas, L. Tian, R. Kattumenu, A. Halim, S. Singamaneni, Freezing the self-assembly process of gold nanocrystals, Chem. Commun. 48 (2012) 1677-1679, https://doi.org/10.1039/c2cc16356j.

[26] A. Onal, Current analytical methods for the determination of biogenic amines in foods, Food Chem. 103 (2007) 1475-1486, https://doi.org/10.1016/j.foodchem. 2006.08.028.

[27] F.M. Salama, K.A. Attia, R.A. Said, A. El-Olemy, A.M. Abdel-Raoof, Disposable gold nanoparticle functionalized and bare screen-printed electrodes for potentiometric determination of trazodone hydrochloride in pure form and pharmaceutical preparations, RSC Adv. 8 (2018) 11517-11527, https://doi.org/10.1039/c8ra00745d.

[28] L.T. Román, Dual enzymatic biosensor for simultaneous amperometric determination of histamine and putrescine, Food Chem. 190 (2016) 818-823, https://doi.org/ 10.1016/j.foodchem.2015.06.035.

[29] W. Henao-escobar, O. Domínguez-renedo, M.J. Arcos-martínez, A screen-printed disposable biosensor for selective determination of putrescine, Microchim Acta. (2013) 687-693, https://doi.org/10.1007/s00604-013-0989-4.

[30] M.A. Alonso-lomillo, O. Domínguez-renedo, P. Matos, M.J. Arcos-martínez, Disposable biosensors for determination of biogenic amines, Anal. Chim. Acta 665 (2010) 26-31, https://doi.org/10.1016/j.aca.2010.03.012.

[31] S. Piermarini, G. Volpe, R. Federico, D. Moscone, G. Palleschi, G. Volpe, R. Federico, D. Moscone, G. Palleschi, G. Palleschi, Detection of biogenic amines in human saliva using a screen-printed biosensor, Anal. Lett. 2719 (2010), https://doi.org/10.1080/ 00032710903518724.

[32] I. Apetrei, C. Apetrei, Amperometric biosensor based on diamine oxidase/platinum nanoparticles/graphene/chitosan modified screen-printed carbon electrode for histamine detection, Sensors 16 (2016) 1-15, https://doi.org/10.3390/s16040422.

[33] C.M. Keow, F.A. Bakar, A.B. Salleh, L.Y. Heng, R. Wagiran, Screen-printed histamine biosensors fabricated from the entrapment of diamine oxidase in a photocured poly (HEMA) film, Electrochem. Sci. 7 (2012) 4702-4715.

[34] S. Pérez, J. Bartrolí, E. Fàbregas, Amperometric biosensor for the determination of histamine in fish samples, Food Chem. 141 (2013) 4066-4072, https://doi.org/10. 1016/j.foodchem.2013.06.125

[35] M. Bhargavi, N. Nesakumar, S. Sethuraman, U. Maheswari, J. Bosco, B. Rayappan, Development of electrochemical biosensor with ceria - PANI core - shell nanointerface for the detection of histamine, Sens. Actuators B. Chem. 199 (2014) 330-338, https://doi.org/10.1016/j.snb.2014.04.009.

[36] Ryosuke Yamada, Nobutaka Fujieda, Maiko Tsutsumi, Seiya Tsujimura, Osamu Shirai, Kenji Kano, Bioelectrochemical determination at histamine dehydrogenase-based electrodes, Electrochemistry 76 (2008) 600-602.

[37] R. Kurita, K. Hayashi, T. Horiuchi, O. Niwa, Differential measurement with a microfluidic device for the highly selective continuous measurement of histamine released from rat basophilic leukemia cells (RBL-2H3), Lab Chip 2 (2002) 34-38, https://doi.org/10.1039/b108803n.

[38] K. Yamamoto, K. Takagi, K. Kano, T. Ikeda, Bioelectrocatalytic detection of histamine using quinohemoprotein amine dehydrogenase and the native electron acceptor cytochrome c-550, Electroanalysis 13 (2001) 375-379.

[39] M. Niculescu, C. Nistor, I. Fre, P. Pec, B. Mattiasson, E. Cso, Redox hydrogel-based amperometric bienzyme electrodes for fish freshness monitoring, Anal. Chem. 72 (2000) 1591-1597.

[40] W. Ye, Y. Xu, L. Zheng, Y. Zhang, M. Yang, P. Sun, A nanoporous alumina membrane based electrochemical biosensor for histamine determination with biofunctionalized magnetic nanoparticles concentration and signal amplification, Sensors 16 (2008), https://doi.org/10.3390/s16101767.

[41] L.E. Delle, C. Huck, S. Grandthyll, K. Jacobs, B. Matthias, P. Wagner, R. Thoelen, M. Weil, R. Lilischkis, X.T. Vu, M.J. Sch, Impedimetric immunosensor for the detection of histamine based on reduced graphene oxide, Phys. Status Solidi Appl. 
Mater. Sci. 1334 (2015) 1327-1334, https://doi.org/10.1002/pssa.201431863.

[42] M. Yang, J. Zhang, X. Chen, Competitive electrochemical immunosensor for the detection of histamine based on horseradish peroxidase initiated deposition of in sulating film, J. Electroanal. Chem. 736 (2015) 88-92, https://doi.org/10.1016/j 